\title{
Vacunación contra influenza para adultos mayores en México: consideraciones económicas
}

Juan Pablo Gutiérrez, M en Economía de la Salud, ${ }^{(1)}$ Stefano M Bertozzi, Dr en Gerencia y Políticas de Salud. $\left..1,2\right)$

\section{Gutiérrez JP, Bertozzi SM. \\ Vacunación contra influenza para adultos mayores en México: consideraciones económicas. Salud Publica Mex 2005;47:234-239.}

Resumen

Objetivo. Presentar una estimación de los costos y resultados de salud que podrían obtenerse en México con la introducción de un programa de vacunación contra influenza en los adultos de 65 años de edad y más. Material y métodos. Entre junio y octubre de 2004, en Cuernavaca, Morelos, México, se elaboró un modelo para estimar el número de años de vida que se pierden por problemas de salud ocasionados por la influenza y el porcentaje de los que podrían salvarse mediante la vacunación por influenza en adultos de 65 años de edad y más, así como el costo asociado a la atención de casos de influenza y el costo potencial de la vacunación, para obtener el costo neto por año de vida salvado. Resultados U tilizando dos escenarios de efectividad de la vacuna, se estimó un total de entre 7454 y 11169 años de vida que podrían salvarse mediante la vacunación de todos los adultos mayores en México a un costo de entre 13301 y 21037 pesos por año. Discusión. Si bien no se comparó con otros usos alternativos de los recursos, la intervención evaluada tiene un costo por año de vida ganado significativamente menor al producto interno bruto per cápita de México, lo que sugiere que es costo efectivo llevar a cabo la vacunación contra influenza en adultos de 65 años de edad en México.

Palabras clave: influenza, análisis de costo-beneficio; análisis económico; vacuna; México
Gutiérrez JP, Bertozzi SM. Influenza vaccination in the elderly population

in Mexico: Economic considerations.

Salud Publica Mex 2005;47:234-239.

\section{A bstract}

Objective. To estimate costs and health outcomes that could be attained by an influenza vaccination program in adults 65 years of age and older in Mexico. Material and methods. Between June and 0 ctober 2004, a model was constructed to estimate the number of life years lost due to influenza and the fraction that could be prevented by vaccination among adults 65 years of age and older. The model also allowed the estimation of the net cost of a vaccination program, including both the cost of delivering the vaccine and savings fro $m$ prevented infections and their treatment costs. Results. Using two scenarios of vaccine effectiveness, between 7454 and 11169 life years could saved by the vaccine if given to all adults 65 years and older in Mexico, with a net cost per life year saved between 13301 and 21037 Mexican pesos (about \$1 210 and \$1 910 US dollars). Discussion. Influenza vaccination among the elderly in Mexico would result in savings per life year saved well below the Mexican gross do mestic product (GDP) per capita, suggesting, even without examining alternative uses for these reso urces, that this is a cost effective inter vention in Mexico and probably also in other middle-income developing countries.

Key words: influenza; cost-benefit analysis; economic analysis; vaccines; M exico

(1) División de Economía y Políticas de la Salud, Centro de Investigación en Sistemas de Salud, Instituto N acional de Salud Pública. Cuernavaca, Morelos, México.

(2) División de Economía, Centro de Investigación y Docencia Económicas, AC (CIDE). México, DF, México.

Fecha de recibido: 21 de octubre de 2004 - Fecha de aprobado: 23 de febrero de 2005

Solicitud de sobretiros: Juan Pablo Gutiérrez. División de Economía y Políticas de la Salud, Centro de Investigación en Sistemas de Salud, Instituto N acional de Salud Pública. Avenida Universidad 655, colonia Santa María Ahuacatitlán 62508 Cuernavaca, Morelos, México. Correo electrónico:jpgutier@insp.mx 
L as infecciones respiratorias agudas (IRA) bajas son la séptima causa de mortalidad, y las enfermedades del sistema respiratorio son la cuarta causa de egresos hospitalarios en los adultos mayores de 65 y más años de edad en México. Durante el año 2001, 7200 personas de este grupo fallecieron de neumonía o sus complicaciones, mientras que durante 2003 cerca de 66000 individuos recibieron atención hospitalaria por enfermedades del sistema respiratorio., ${ }^{1,2}$

El virus de la influenza es una de las principales causas de enfermedades respiratorias agudas en todos los grupos de edad, que afecta más severamente en los extremos de la vida. Las tasas de infección más altas ocurren durante la infancia, pero las tasas de casos graves y de mortalidad debido a la influenza tienen su punto más alto entre los adultos de 65 años de edad y más. ${ }^{3}$

Del total de IRA entre los adultos mayores, se estima que entre 10 y $15 \%$ son ocasionadas por el virus de la influenza, con una tasa de letalidad similar o mayor al promedio de todas las IRA. ${ }^{4}$

La vacunación contra el virus de la influenza se ha propuesto como una estrategia para reducir las consecuencias en muertes y hospitalizaciones. Debido a las características de mutación del virus, la vacuna es efectiva para las cepas presentes en el año de vacunación, y debe ser reformulada cada año. Ello implica que es una vacuna de aplicación anual, que previene potencialmente sólo los casos de la temporada en la que se emplea. ${ }^{5}$

El propósito de este análisis es presentar una estimación de los costos y resultados de salud que podrían obtenerse en México a través de la vacunación contra influenza en los adultos de 65 años de edad y más.

\section{Material y métodos}

Entre junio y octubre de 2004 se elaboró un modelo para estimar, por un lado, el número de muertes y egresos hospitalarios entre los adultos de 65 años de edad o más, que se evitarían en el país con una campaña de vacunación con cobertura universal; y por el otro, los costos asociados con la atención médica en que se hubiera incurrido de no evitarse los mismos casos, los que entonces se asumen como los ahorros por la vacunación.

Para la estimación del número de muertes y egresos evitados se utilizaron las estadísticas reportadas por la Secretaría de Salud (SSA) para el número de muertos y egresos hospitalarios por neumonía e influenza durante 2001 y 2003, respectivamente, ${ }^{1,2}$ los cuales se combinaron con estimaciones del porcentaje de muertes y casos que podrían evitarse con la efectividad conocida de la vacuna, considerando el porcen- taje de neumonías que son ocasionadas por el virus de la influenza, y la eficacia de la vacuna. Debido a la incertidumbre en el porcentaje de muertes evitadas, se estimaron simultáneamente dos escenarios (uno que asume $30 \%$ de muertes evitadas con la vacuna y otro que reduce este porcentaje a $20 \%$ ). En el cuadro I se presentan los valores de las variables empleadas para la construcción del modelo y sus fuentes.

Para el número de años de vida perdidos por influenza, se usaron datos del Consejo Nacional de Población (Conapo) sobre la probabilidad de muerte para cada año de edad; con esto se estimó la esperanza de vida por año de edad de 65 a 100 años. ${ }^{7}$ Asimismo, utilizando el número de egresos y el peso de discapacidad notificados por la Organización Mundial de la Salud (OMS) para casos de neumonía, se estimó el número de años perdidos por discapacidad por neumonía. ${ }^{8}$

Además, con base en la información de la SSA sobre costos de atención de casos de neumonía ${ }^{9}$ y costo por dosis de la vacuna, se estimaron tanto los ahorros potenciales por los casos evitados, como el costo de la cobertura universal. De este modo, se estimó el costo neto de la vacunación (costo de aplicación del biológico menos los ahorros por muertes y egresos evitados). También se utilizaron datos de otro estudio para el costo de aplicación por dosis, así como transporte y almacenamiento.*

Debido a que la vacuna debe aplicarse cada año, los costos de aplicación son anuales, mientras que los años potencialmente ganados ocurren en un horizonte temporal de hasta 12 años. Por ello, la esperanza de vida se descontó con una tasa de $3 \%$ anual para tener el número de años en valor presente.

Finalmente, con la información anteriormente descrita se estimó el costo neto por año de vida potencialmente ganado a través de la vacunación universal de los adultos en el grupo de edad ya señalado.

Para los dos escenarios descritos arriba, se llevó a cabo un análisis de sensibilidad probabilístico para permitir variación en los costos de atención, la eficacia de la vacuna y la cobertura real de la vacunación. En el cuadro II se presentan los rangos y tipos de distribución para cada variable utilizados para el análisis de sensibilidad. En el caso de la cobertura, se supuso una distribución triangular con $100 \%$ como el valor más probable (y máximo) y $60 \%$ como el menor, conside-

\footnotetext{
* Colchero A, Gutiérrez JP, Bertozzi S. Cost effectiveness of Uniject versus the traditional syringe and vial method in vaccination campaigns. Cuernavaca, Morelos: Instituto Nacional de Salud Pública; 2002. Documento no publicado.
} 
Cuadro I

\section{Parámetros y valores incluidos en el modelo para estimar costos de la vacunación contra influenza EN ADULtos de 65 años de EdAD Y MÁs. MÉXICO, 2004}

\begin{tabular}{|c|c|c|}
\hline Parámetro & Valor & Fuente \\
\hline $\begin{array}{l}\text { Total de muertes por neumonía e influenza en adultos de } 65 \text { años } \\
\text { de edad y más en } 2001\end{array}$ & 6753 & Secretaría de Salud \\
\hline $\begin{array}{l}\text { Egresos por neumonía e influenza en adultos de } 65 \text { años de edad } \\
\text { y más (excluyendo egresos por muerte) en } 2003\end{array}$ & 12951 & Secretaría de Salud ${ }^{6}$ \\
\hline Población total de 65 años de edad y más para 2004 & 5.42 millones & Conapo $^{1}$ \\
\hline Esperanza de vida para las personas de 65 años de edad y más & $\begin{array}{l}\text { De } 12.8 \text { años para mujeres de } 65 \text { años } \\
\text { a } 0.5 \text { años para hombres de } 100 \text { años } \\
\text { de edad o más }\end{array}$ & $\begin{array}{l}\text { Conapo }^{7} \text { (probabilidades } \\
\text { de muerte) y cálculos } \\
\text { de los autores }\end{array}$ \\
\hline Discapacidad por neumonía & 0.280 & OMS \\
\hline Duración promedio de un episodio de neumonía o influenza & 12.7 días & Secretaría de Salud, ajuste de los autores \\
\hline Eficacia de la vacuna para evitar muertes & $\begin{array}{l}30 \% \text { (Escenario 1) } \\
20 \% \text { (Escenario 2) }\end{array}$ & Literatura \\
\hline Eficacia de la vacuna para evitar casos & $\begin{array}{l}20 \% \text { (Escenario 1) } \\
20 \% \text { (Escenario 2) }\end{array}$ & Literatura $^{3}$ \\
\hline Costo del biológico por dosis & $35^{*}$ & Secretaría de Salud \\
\hline Costo de transporte y almacenamiento por dosis & $0.3^{*}$ & Colchero et al. \\
\hline Costo de aplicación por dosis & $2.5^{*}$ & Colchero et al. \\
\hline Costo de hospitalización por neumonía & 12183 & Secretaría de Salud \\
\hline
\end{tabular}

Cuadro II

\section{Parámetros y rangos utilizados para el anÁlisis DE SENSIBILIDAD PROBABILÍSTICO DE LOS VALORES DE COSTOS Y EFECTIVIDAD DE LA VACUNACIÓN CONTRA INFLUENZA EN ADULTOS DE 65 AÑOS DE EDAD Y MÁS. MÉxıco, 2004}

\begin{tabular}{|c|c|c|}
\hline Parámetro & Rango & Distribución \\
\hline Cobertura de vacunación & 60 a $100 \%$ & Triangular \\
\hline Eficacia de la vacuna para evitar muertes & $\begin{array}{c}20 \text { a } 40 \% \\
\text { (Escenario } 1 \text { ) } \\
10 \text { a } 30 \% \\
\text { (Escenario 2) }\end{array}$ & Uniforme \\
\hline Eficacia de la vacuna para evitar casos & $\begin{array}{c}10 \text { a } 30 \% \\
\text { (Escenario } 1) \\
10 \text { a } 30 \% \\
\text { (Escenario 2) }\end{array}$ & Uniforme \\
\hline Costo de hospitalización por neumonía & 7342 & Exponencial \\
\hline
\end{tabular}

rando las altas coberturas alcanzadas en México para otras vacunas. Para la eficacia de la vacunas, y debido a la falta de más fuentes de información, se propuso una distribución uniforme con 10 puntos porcentuales por debajo y por arriba del valor más probable. Final- mente, para la distribución de los costos se empleó una distribución exponencial basada en datos de otros estudios. Para el análisis de sensibilidad se realizaron 100 mil iteraciones del modelo.

Asimismo, para ambos escenarios se estimaron los resultados modificando la tasa de descuento de 3 a $0 \%$, 5 y $10 \%$. La tasa de $3 \%$ es la recomendada en la literatura universal, y utilizar una tasa de $0 \%$ es asumir que otorgamos el mismo valor a los años en el futuro que a los años en el presente. Tomando en cuenta que la tasa de descuento social se encuentra inversamente correlacionada con el nivel de desarrollo, se esperaría que ésta fuera mayor para países menos desarrollados en donde las necesidades presentes hacen más fuerte la preferencia por beneficios actuales, en comparación con beneficios en el futuro.

\section{Resultados}

Los resultados de las estimaciones se presentan en el cuadro III. Como puede observarse, el número de años que potencialmente se ganarían con la vacunación depende sobre todo de las muertes evitadas, y muy poco de la discapacidad. Esto se debe en buena medida a la recuperación casi total, en relativamente poco tiempo, de la gran mayoría de personas que no mueren en la fase aguda del padecimiento. 
La magnitud de los ahorros potenciales representa aproximadamente entre 23 y $27 \%$ del costo de la vacunación, según el escenario que se considere. De esta forma, la vacunación representa un costo positivo.

Por lo que se refiere al costo por año de vida con calidad ganado, la estimación conforme a los escenarios modelados se encuentra entre 13 mil y 21 mil pesos mexicanos de 2004.

Por otra parte, si se separan los resultados por subgrupos de edad (cuadro III), el costo es significativamente menor para el grupo de 80 años y más, en donde el costo por año ganado es únicamente entre 13 y $19 \%$ del valor para el grupo completo. Esto se debe a que los ahorros se concentran en este subgrupo: porcentualmente, para los adultos de 80 años de edad y más, los ahorros representan alrededor de $50 \%$ del costo de la vacunación, lo que es una consecuencia de una mayor incidencia de hospitalizaciones y muertes en este grupo de edad.

Los resultados del análisis de sensibilidad se destacan en el cuadro IV. La variable para la que los resultados son más sensibles es la eficacia de la vacuna para evitar muertes, seguida del costo por hospitalización. Los valores medios que resultan del análisis de sensibilidad se mantienen dentro de los límites de lo que parece socialmente aceptable, y esto se sostiene para $95 \%$ de las iteraciones realizadas. En la figura 1 se presentan la distribución y los rangos del análisis de sensibilidad.

Asimismo, en el último cuadro se presenta el costo por año de vida ajustado por discapacidad para ambos escenarios y se asumen distintas tasas de descuento. En el valor más bajo, sin descuento, el resultado es
$11 \%$ menor al modelo base, mientras que con la tasa más alta, de $10 \%$, el resultado es $28 \%$ mayor al modelo base. Aun en este incremento el costo por año de vida salvado se encuentra por debajo del producto interno bruto (PIB) per cápita.

\section{Discusión}

Los resultados muestran que la vacunación contra influenza en adultos de 65 años de edad y más en México tiene costos positivos, por lo que su implementación debe considerarse en un contexto de intervenciones alternativas, para lo cual es importante comparar el costo por unidad de resultado obtenida.

Las estimaciones indican que el costo por año de vida potencialmente ganado se ubica entre los $12 \mathrm{mil}$ y 20 mil pesos. La Comisión de Macroeconomía y Salud ha sugerido que las intervenciones con un costo menor a tres veces el PIB per cápita por año de vida ganado representan una buena inversión. ${ }^{9}$ Por su lado, la OMS, en su informe de 2002, establece que una intervención es muy costo efectiva si cuesta menos del PIB per cápita por año salvado, y costo-efectiva si se paga entre una y tres veces el PIB per cápita por año de vida salvado. ${ }^{10}$ En este contexto, la intervención es claramente aceptable.

Aunado al resultado del modelo base, el análisis de sensibilidad, así como las variaciones en la tasa de descuento, muestran que el resultado es robusto en el sentido de un costo por año de vida ajustado por discapacidad salvado menor al PIB per cápita. En el escenario más pesimista para la vacuna, el costo es menos de la mitad del PIB per cápita.

\section{ESTIMACIONES DE COSTOS, EFECTIVIDAD Y COSTO POR AÑO DE VIDA AJUSTADO POR DISCAPACIDAD SALVADO POR LA VACUNACIÓN CONTRA INFLUENZA EN ADULTOS DE 65 AÑOS Y MÁS, DE ACUERDO CON LOS ESCENARIOS PLANTEADOS. MéXICO, 2004}

Variable de resultado

Años de vida perdidos por muertes debidas al virus de la influenza

Años de vida saludable perdidos por morbilidad debida a infecciones de virus de influenza

Años totales salvados por la vacuna

Costo neto de la vacunación

Costo por año de vida ajustado por discapacidad salvado (tasa de descuento base, 3\%)

Subgrupos (escenario 1)

Años de vida ajustados por discapacidad totales salvados por la vacuna

Costo neto de la vacunación

Costo por año saludable salvado

* Pesos mexicanos

Estimación de los autores. Cuernavaca, 2004
Escenario 1

Escenario 2

37272

126

11169

149 mill.* 157 mill.*

13 303* $21037^{*}$

\begin{tabular}{cccc}
\multicolumn{2}{c}{65 y más } & 70 y más & \multicolumn{2}{c}{75 y más } & 80 y más \\
\hline 11 169 & 9642 & 7846 & 5810 \\
\hline 149 mill.* & 82 mill.* & 37 mill.* & 10 mill.* \\
\hline 13 303* & $8546^{*}$ & $4697^{*}$ & $1720^{*}$
\end{tabular}


Cuadro IV

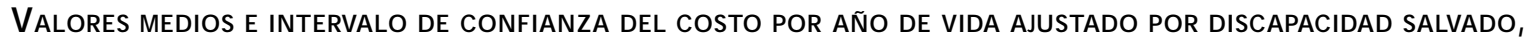 POR GRUPO DE EDAD Y ESCENARIO, DE ACUERDO CON EL ANÁLISIS DE SENSIBILIDAD PROBABILÍSTICA Y ESTIMACIONES DE COSTO POR AÑo DE VIDA SALVAdo PARA DISTINTAS TASAS DE DESCUENTO. MÉXICO, 2004*}

Análisis de sensibilidad probabilístico

Variable de resultado

Escenario 1

Media

$(5 \%, 95 \%)$

Escenario 2

Media

$(5 \%, 95 \%)$

Costo por año salvado, 65 años de edad y más

18622

30421

(9 591, 28 848)

Costo por año salvado, 70 años de edad y más

12874

(4 656, 20 959)

Costo por año salvado, 75 años de edad y más

8221

$(15076,53608)$

21381

$(471,14668)$

4676

$604,38986)$

Costo por año salvado, 80 años de edad y más

$(<0,10060)$

030,27293)

Escenario $1 \quad$ Escenario 2

Variaciones en tasa de descuento

Variable de resultado

\begin{tabular}{ll}
13303 & 21037 \\
11787 & 18639 \\
\hline 14350 & 22690 \\
17081 & 26999
\end{tabular}

Costo por año de vida ajustado por discapacidad salvado (tasa de descuento base, $3 \%$ )

Costo por año de vida ajustado por discapacidad salvado (tasa de descuento $=0 \%$ )

Costo por año de vida ajustado por discapacidad salvado (tasa de descuento $=5 \%$ )

Costo por año de vida ajustado por discapacidad salvado (tasa de descuento $=10 \%$ )

17081

26999

* En pesos mexicanos

Estimación de los autores. Cuernavaca, 2004

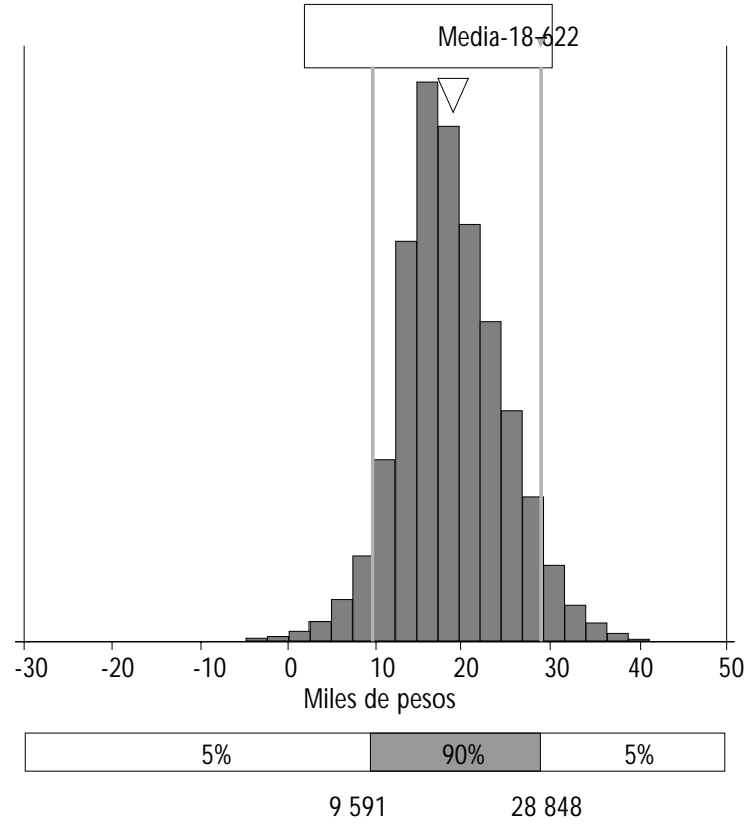

Figura 1. Distribución del COSTO POR AÑo de VIDA AJUSTADO POR DISCAPACIDAD GANADO CON LA VACUNACIÓN POR INFLUENZA EN LOS ADULTOS DE 65 AÑOS DE EDAD Y MÁS, ESTIMADO EN 100 MIL ITERACIONES, DE ACUERDO CON LOS VALORES DEL ANÁLISIS DE SENSIBILIDAD
Sin embargo, es importante notar que las estimaciones presentadas se basan en un grupo de supuestos: por un lado, los costos para la vacunación no necesariamente incluyen todos los insumos necesarios, además que parten del supuesto que no es necesario crear un mecanismo alterno para la provisión del servicio sino que se monta sobre las campañas y sistemas ya existentes de vacunación, lo que podría estar subestimando el costo real. Si bien se puede asumir que no es necesario un mecanismo alternativo para administrar la vacuna, ciertos costos operativos seguramente sí serán indispensables.

Por otra parte, se asume una alta cobertura de la vacuna, lo que requiere de una alta aceptación de la misma. Diversos estudios han sugerido que entre los adultos mayores la tasa de aceptación para intervenciones nuevas es menor que en otros grupos de edad, lo que implicaría que, para alcanzar una alta tasa de cobertura, habría que considerar costos adicionales para una campaña de promoción.

No obstante, los resultados de este ejercicio sugieren que la vacunación de adultos mayores contra influenza es económicamente justificable, si bien es necesario desarrollar estudios que reduzcan la incertidumbre en las estimaciones y ofrezcan un costo por año más preciso. 


\section{Referencias}

1. Secretaría de Salud. Principales causas de mortalidad en edad posproductiva (65 años y más) 2001. Sistema $\mathrm{N}$ acional de Información en Salud. Disponible en: http://www.salud.gob.mx/apps/htdocs/ estadisticas/egresoshosp/danossalud.htm [2004 junio 17]. 2. Secretaría de Salud. Sistema N acional de Vigilancia Epidemiológica, Epidemio logía, Semana 16, México, D F: Secretaría de Salud; 2004. 3. Harper SA, Fukuda K, Uyeki TM, C ox N J, Bridges C B. Centers for Disease Control and Prevention (CDC) Advisory Committee on Immunization Practices (ACIP). Prevention and control of influenza: Recommendations of the Advisory Committee on Immunization Practices (ACIP). MMW R Morb Mortal W kly Rep Recomm Rep 2004 May 28:53(RR-6):1-40.

4. Ayora-Talavera G, Góngora-Biachi RA, López-Martínez I, MoguelRodríguez W, Pérez-C arrillo H,Vázquez-ZapataV et al. D etection of human influenza virus in Yucatan, Mexico. Rev Invest Clin 2002; 54(5):410-414.

5.W orld Health 0 rganization. Influenza vaccines:W HO position paper: W kly Epidemiol Rec 2002;77:230-240.
6. Estadística de egresos hospitalarios del sector público del Sistema N acional de Salud, 2003. Salud Publica Mex 2004; 46(5):464-487.

7. Consejo $\mathrm{N}$ acional de Población. Proyecciones de la población de México, 2000-2050. México, DF: Conapo; 2002.

8. Murray CJL, López,AD, ed.The global burden of disease:A comprehensive assessment of mortality and disability from diseases, injuries and risk factors in 1990 and projected to 2020. Cambridge (MA): Harvard University Press; 1996. (Global Burden of Disease and Injury Series, Vol. 1).

9. Lozano AR, Gómez FS, Herrera BE, Sousa FA, Avila-Figuero C. Estimación de los costos de producción de servicios clínicos para la prevención, diagnóstico y tratamiento médico en el sistema de salud mexicano. México, DF: Secretaría de Salud; 2003.

10.W orld Health 0 rganization. Comission. Macroeconomics and health: Investing in health for economic development. Report of the Comission on Macroeconomics and health. Ginebra:W orld Health 0 rganization; 2001.

11. W orld Health 0 rganization. The W orld Health Report 2002 Reducing risks, promoting healthy life. G inebra:W orld Health O rganization; 2002. 